\title{
Delayed Onset of Acute Limb Compartment Syndrome With Neuropathy After Venoarterial Extracorporeal Membrane Oxygenation Therapy
}

\author{
Jin Young Go, $\mathrm{MD}^{1}$, Yu-Sun Min, $\mathrm{MD}^{2}$, Tae-Du Jung, $\mathrm{MD}^{1,2}$ \\ ${ }^{1}$ Department of Rehabilitation Medicine, Kyungpook National University College of Medicine, Daegu; \\ ${ }^{2}$ Department of Rehabilitation Medicine, Kyungpook National University Hospital, Daegu, Korea
}

\begin{abstract}
Acute limb compartment syndrome (ALCS) is defined as compound symptoms resulting from poor oxygenation and decreased nutrition supply to muscles and nerves in a tightly confined compartment. The most common cause of ALCS is tibia fracture, followed by blunt trauma to soft tissue. However, non-traumatic causes are rare. We report an iatrogenic, non-traumatic ALCS case after venoarterial extracorporeal membrane oxygen (VA-ECMO) therapy. A 14-year-old male received VA-ECMO therapy due to cardiorespiratory failure after drowning. Although he had no symptoms during therapy, leg swelling appeared 10 hours after ECMO treatment. Two days after the leg swelling, the patient underwent a fasciotomy. Unfortunately, nerve conduction studies and electromyography showed multiple neuropathies in the lower leg. Despite 2 weeks of rehabilitation with electrical stimulation, an exercise program, and physical therapy, there was no definite change in muscle strength. To our knowledge, this is the first reported case of non-traumatic ALCS after VA-ECMO therapy in Korea.
\end{abstract}

Keywords Anterior compartment syndrome, Extracorporeal membrane oxygenation

\section{INTRODUCTION}

Acute limb compartment syndrome (ALCS) is a surgical emergency. Delayed diagnosis and treatment can lead to permanent dysfunction in the limb involved [1]. A tibia fracture is the most common cause, followed by blunt

Received May 28, 2013; Accepted September 17, 2013

Corresponding author: Tae-Du Jung

Department of Rehabilitation Medicine, Kyungpook National University College of Medicine, 130 Dongdeok-ro, Jung-gu, Daegu 700-721, Korea Tel: +82-53-200-5311, Fax: +82-53-423-0389, E-mail: teeed0522@hanmail. net

(c) This is an open-access article distributed under the terms of the Creative Commons Attribution Non-Commercial License (http://creativecommons. org/licenses/by-nc/3.0) which permits unrestricted noncommercial use, distribution, and reproduction in any medium, provided the original work is properly cited.

Copyright $\odot 2014$ by Korean Academy of Rehabilitation Medicine trauma to soft tissue [2]. Non-traumatic vascular injury is an extremely rare cause of ALCS. This paper focuses on ALCS due to ECMO therapy, which has not been reported previously in Korea, and only rarely worldwide.

\section{CASE REPORT}

A 14-year-old male suffered cardiac arrest after drowning. His circulation was restored after $\mathbf{3 0}$ minutes of cardiopulmonary cerebral resuscitation. The subject was under mechanical ventilation after admission to a local medical center, but was transferred to the emergency room due to progressing pulmonary edema.

His initial blood pressure was $57 / 27 \mathrm{mmHg}$ and body temperature was $36^{\circ} \mathrm{C}$. On arterial blood gas analysis, $\mathrm{pH}$ was 7.023, arterial oxygen pressure $\left(\mathrm{PaO}_{2}\right) 22.3 \mathrm{mmHg}$, 
and oxygen saturation $19.7 \%$. His carbon dioxide pressure $\left(\mathrm{PCO}_{2}\right)$ was $56.2 \mathrm{mmHg}$, and he showed respiratory acidosis. The patient's elevated levels myoglobin 1,300 $\mathrm{ng} / \mathrm{mL}$, CK-MB $22.8 \mathrm{ng} / \mathrm{mL}$, and creatine phosphokinase $1,120 \mathrm{U} / \mathrm{L}$, were consistent with rhabdomyolysis; a result from hypothermia and cardiogenic shock.

VA-ECMO therapy was started with the diagnosis of cardiorespiratory failure and rhabdomyolysis. Catheters (21 and $19 \mathrm{Fr}$ ) were inserted in the right femoral vein and artery, respectively. VA-ECMO had been applied for 38 hours at $1,500-2,500 \mathrm{rpm}, \mathrm{FiO}_{2}$ of $0.7-0.8$, and a pump flow of 4.0-6.0 L/min. During ECMO therapy, the patient was sedated (Glasgow Coma Scale score of $<5$ ), receiving mechanical ventilation and therefore could not express any pain or discomfort.

During 38 hours of ECMO therapy, the pulse in all four limbs were weak and both systolic and diastolic blood pressures were low, without asymmetry. The patient showed no signs that would indicate ALCS (i.e., limb swelling, cyanosis, or decreased pulse pressure). However, 10 hours after ECMO disconnection, swelling of the right calf muscle was apparent. Sixteen hours after ECMO disconnection, the pulse in the right dorsalis pedis artery was weaker than that of the left side, and cyanosis developed in the foot dorsum. We obtained X-ray images, serum D-dimer levels and duplex ultrasonograms to rule out a tibial fracture and deep vein thrombosis. There was no definite abnormal finding in the tibia or ankle X-ray. Despite a slight increase in D-dimer level $(4.36 \mathrm{mg} / \mathrm{L})$, duplex ultrasonography of the right common femoral vein, superficial femoral vein and popliteal vein showed no evidence of deep vein thrombosis. Ultrasonograms showed prominent edematous changes in the right calf muscle (Fig. 1). Levels of neutrophils, Creactive protein, myoglobin, and aspartate aminotransferase (AST) were elevated in laboratory tests (Fig. 2). Due to aggravation of
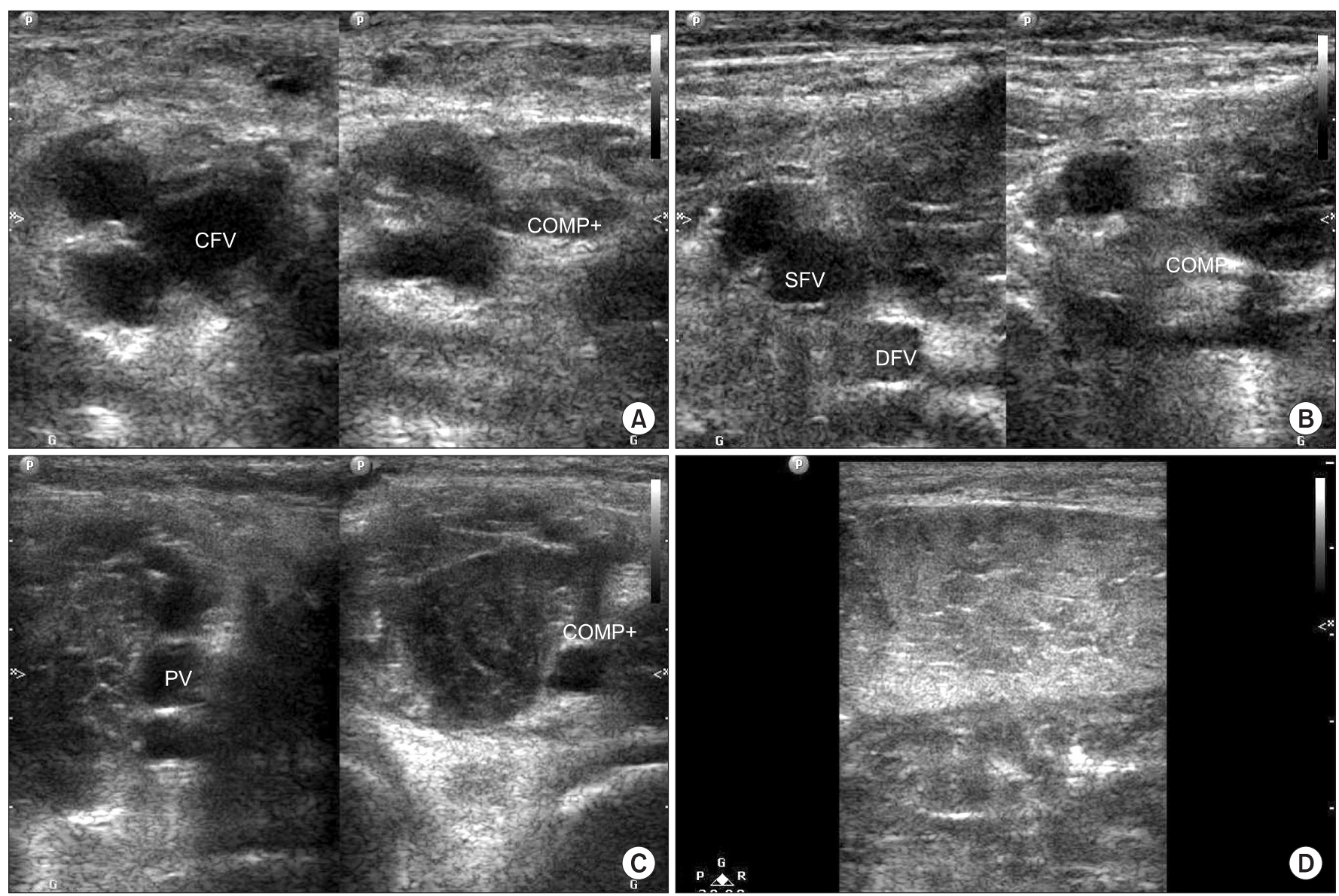

Fig. 1. Duplex ultrasonography performed in right leg. (A) The right common femoral vein (CFV); (B) deep femoral vein (DFV) and superficial femoral vein (SFV); and (C) popliteal vein (PV) is compressed by leg swelling (D). Ultrasonography showed no evidence of deep vein thrombosis. COMP, compression. 
(A)

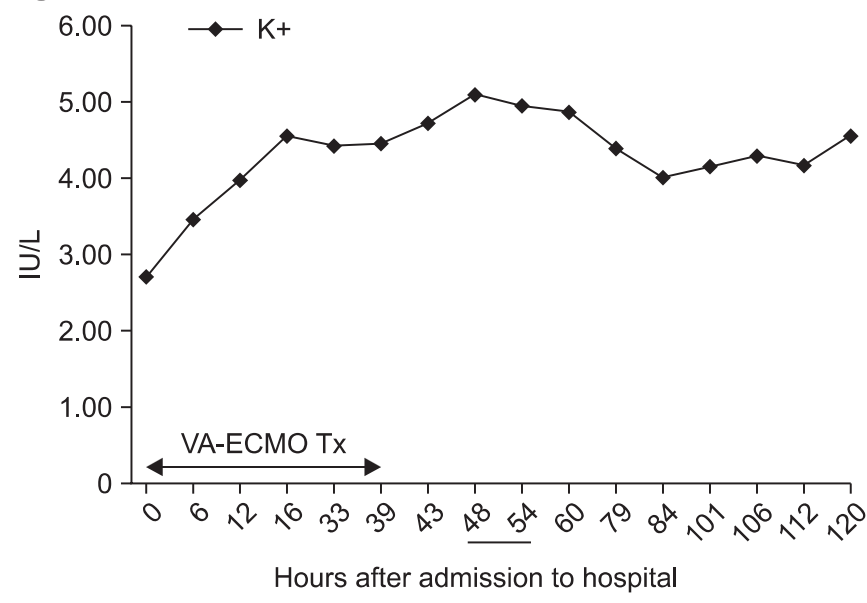

(C)

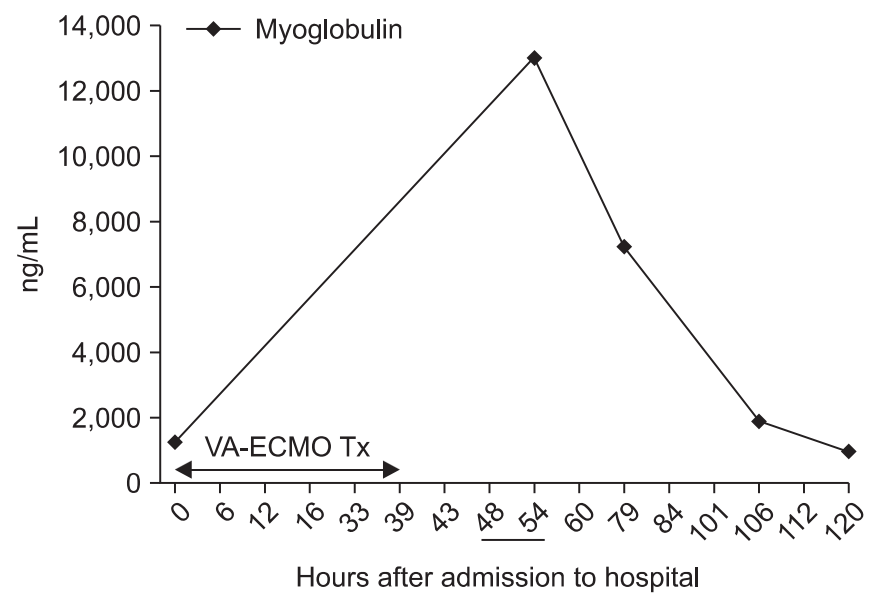

symptoms, a fasciotomy was performed on the anterolateral and posteromedial sides two days after leg swelling. Four days after the fasciotomy, the pulse in the right foot dorsum became stronger and the cyanosis improved.

Due to persistent right lower limb weakness, the patient was referred to the Department of Rehabilitation Medicine one month after admission. We conducted a manual muscle test (MMT) and performed electromyography (EMG). MMT determined his motor power was $3 / 5$ in the unaffected limb, and hip flexor $3 / 5$, knee extensor $3 / 5$, ankle dorsiflexor $1 / 5$, great toe extensor $1 / 5$, and ankle plantar flexor $1 / 5$ in the affected limb. Motor nerve conduction studies showed no response in the right peroneal or tibial nerves. A sensory nerve conduction study showed no response in the right superficial peroneal or sural nerves (Table 1). Needle EMG showed abnormal spontaneous activity on right tibialis anterior, tibialis

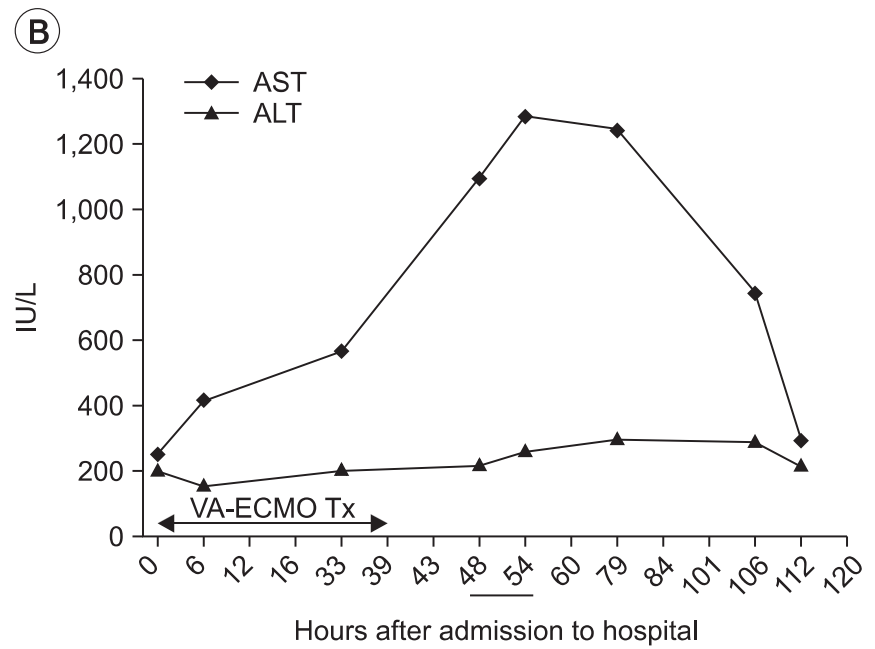

Fig. 2. Chronological change of potassium ion (A), AST and ALT (B), and myoglobulin (C) after venoarterial extracorporeal membrane oxygenation treatment (VA-ECMO Tx). It shows peak values when the right leg swelling are observed, about 10-16 hours after ECMO disconnection (underlined). $\mathrm{K+}$, potassium; AST, aspartate aminotransferase; ALT, alanine aminotransferase.

posterior, peroneus longus (PL), and the medial head of the gastrocnemius (GCN). GCN and PL muscles showed polyphasic motor unit action potentials with large amplitudes and long duration (Table 2). The patient was diagnosed with right peroneal, tibial, sural, and superficial peroneal neuropathy based on the EMG findings, which is consistent with compartment syndrome. The patient was discharged after 2 weeks of rehabilitation including electrical stimulation, strengthening exercise, and gait training. At discharge, there was no definite improvement in muscle strength.

\section{DISCUSSION}

ALCS is defined as compound symptoms showing elevated pressure and microcirculation impairment resulting from poor oxygenation and nutrition supply to mus- 
Table 1. Initial findings of nerve conduction studies in the both lower extremities

\begin{tabular}{|c|c|c|c|c|c|}
\hline Nerve & $\begin{array}{c}\text { Stimulation } \\
\text { site }\end{array}$ & $\begin{array}{c}\text { Recording } \\
\text { site }\end{array}$ & $\begin{array}{c}\text { Latency } \\
(\mathrm{ms})\end{array}$ & $\begin{array}{l}\text { Amplitude } \\
(\mu \mathrm{c})\end{array}$ & $\begin{array}{c}\text { Velocity } \\
(\mathrm{m} / \mathrm{s})\end{array}$ \\
\hline \multicolumn{6}{|l|}{ Motor } \\
\hline \multirow[t]{2}{*}{ Rt. peroneal } & Ankle & EDB & NR & - & - \\
\hline & Knee & - & NR & - & - \\
\hline \multirow[t]{2}{*}{ Lt. peroneal } & Ankle & EDB & 3.3 & 5.4 & - \\
\hline & Knee & - & 10.6 & 3.6 & 45.2 \\
\hline \multirow[t]{2}{*}{ Rt. tibial } & Ankle & $\mathrm{AH}$ & NR & - & - \\
\hline & Knee & - & NR & - & - \\
\hline \multirow[t]{2}{*}{ Lt. tibial } & Ankle & $\mathrm{AH}$ & 3.05 & 11.4 & - \\
\hline & Knee & - & 11.7 & 8.9 & 46.2 \\
\hline \multicolumn{6}{|l|}{ Sensory } \\
\hline Rt. superficial peroneal & Lateral leg & Medial to LM & NR & - & - \\
\hline Lt. superficial peroneal & Lateral leg & Medial to LM & 2.7 & 11 & - \\
\hline Rt. sural & Calf & Posterior to LM & NR & - & - \\
\hline Lt. sural & Calf & Posterior to LM & 3.35 & 22.2 & - \\
\hline
\end{tabular}

Rt., right; Lt., left; EDB, extensor digitorum brevis; AH, abductor hallucis; NR, no response; LM, lateral malleolus.

Table 2. Initial findings of needle electromyography

\begin{tabular}{|c|c|c|c|c|c|c|}
\hline \multirow{2}{*}{ Muscle } & \multicolumn{2}{|c|}{ Spontaneous activities } & \multicolumn{3}{|c|}{ MUAP } & \multirow{2}{*}{ Recruit } \\
\hline & Fib & PSW & Polyphasic & Amplitude & Duration & \\
\hline Rt. vastus medialis & None & None & Normal & Normal & Normal & Normal \\
\hline Rt. semimembranosus & None & None & Normal & Normal & Normal & Normal \\
\hline Rt. biceps femoris (short head) & None & None & Normal & Normal & Normal & Normal \\
\hline Rt. gastrocnemius (medial head) & $1+$ & $1+$ & $\uparrow$ & Large & Long & $\mathrm{R}$ \\
\hline Rt. tibialis anterior & $1+$ & $1+$ & Abs & Abs & Abs & Abs \\
\hline Rt. tibialis posterior & $1+$ & $3+$ & Abs & Abs & Abs & Abs \\
\hline Rt. peroneus longus & $1+$ & $3+$ & $\uparrow$ & Large & Long & $\mathrm{R}$ \\
\hline Rt. L3 PSP & None & None & & & & \\
\hline Rt. L4 PSP & None & None & & & & \\
\hline Rt. L5 PSP & None & None & & & & \\
\hline Rt. S1 PSP & None & None & & & & \\
\hline
\end{tabular}

Rt., right; MUAP, motor unit action potential; Fib, fibrillation; PSW, positive sharp wave; R, reduced recruitment; Abs, absent; $\uparrow$, increased; PSP, paraspinal muscle.

cles and nerves in a tightly confined compartment $[3,4]$. Generally, this diagnosis is made by clinical findings, although intracompartmental pressure (ICP) monitoring, magnetic resonance imaging, and ultrasonography are used to supplement this. The main symptoms of ALCS are pain, paresthesia, dysesthesia, weakness, and pulselessness [4].

This case of ALCS may be explained by the ischemiareperfusion mechanism [5]. The first phase is an isch- emic phase. As in previous case reports regarding ALCS after ECMO therapy [6], 19- and 21-Fr large-bore cannulas without backflow ports (allowing flow to the limb through the cannula) were inserted in the femoral artery and vein, respectively. Because these cannulas occupy a relatively large proportion of the vascular area, arterial blood pressure is reduced and venous blood pressure is increased $[3,6]$. By the arteriovenous gradient theory, a reduced arteriovenous gradient decreases the blood sup- 
ply to the tissue [7]. As a result of ischemia, hypoxic damage of the vascular endothelium induces leukocytosis, releasing inflammatory mediators and eventually causing a vicious cycle of microcirculation impairment [7]. At the same time, expression of proinflammatory gene products are increased and also white blood cells are migrated from intravascular space to interstitium. Thus this phase is regarded as a proinflammatory state, where risk of further reperfusion injury is increased [5]. The second phase is reperfusion phase. During the ECMO weaning period, reperfusion to ischemic tissue occurs. Reactive oxygen species and enzyme cofactors are carried to the ischemic tissues through the blood stream. Inflammatory cells in the interstitium, such as white blood cells and platelets which migrated at ischemic phase, reduce vascular permeability. The no-reflow phenomenon can occur in this period. The no-reflow phenomenon refers to the clinical observation that blood flow is not fully restored after reperfusion. Possible mechanisms include increased platelet-leukocyte aggregation, and interstitial fluid accumulation, these are manifested clinically as target organ damage $[5,8]$. We think that the no-reflow phenomenon is possibly the most important mechanism in ALCS after VA-ECMO treatment. In this ALCS case, the elevation curves in serial laboratory tests of potassium, AST, and myoglobulin are reflected as cell death and skeletal muscle necrosis, provides evidence supporting the ischemiareperfusion mechanism of ALCS (Fig. 2). Potassium, AST, and myoglobin showed peak values at about 10-16 hours after ECMO disconnection (Fig. 2). This indicates the tissue injury of acute compartment syndrome occurred after reperfusion [9]. By clinical course and laboratory findings, we can strongly assert that ALCS was caused by the ECMO therapy. However, in order to prove the pathophysiology mentioned above, continuous vascular pressure monitoring will be needed in further studies.

For early diagnosis, ICP monitoring is one of the most important objective findings [4]. It is especially useful in patients with altered consciousness. In this case, the medical staff did not recognize the risk of ALCS and therefore the ICP was not monitored; therefore, the diagnosis of ALCS was delayed even more. Although longterm follow-up will be needed, some neurological sequalae will remain. This agrees with previous reports that recovery of normal function in ALCS subjects who do not undergo a fasciotomy within 12 hours is rare $[6,10]$.
We reported the pathophysiologic findings in delayedonset ALCS with neuropathy during VA-ECMO therapy. Tissue ischemia can begin at the beginning of ECMO treatment, but can be overlooked without careful observation. Once symptoms occur by ischemia-reperfusion cascade, serious problems, such as paralysis, multi-organ failure and even death, can result without immediate treatment. However, with early detection, anti-inflammatory, anti-thrombotic and immunologic therapies can prevent catastrophic sequalae [5]. As was demonstrated in this case, serum lab findings and the clinical findings of the patient are helpful in early detection.

\section{CONFLICT OF INTEREST}

No potential conflict of interest relevant to this article was reported.

\section{REFERENCES}

1. Malik AA, Khan WS, Chaudhry A, Ihsan M, Cullen NP. Acute compartment syndrome: a life and limb threatening surgical emergency. J Perioper Pract 2009;19: 137-42.

2. McQueen MM, Gaston P, Court-Brown CM. Acute compartment syndrome. Who is at risk? J Bone Joint Surg Br 2000;82:200-3.

3. Moore RE 3rd, Friedman RJ. Current concepts in pathophysiology and diagnosis of compartment syndromes. J Emerg Med 1989;7:657-62.

4. Elliott KG, Johnstone AJ. Diagnosing acute compartment syndrome. J Bone Joint Surg Br 2003;85:625-32.

5. Eltzschig HK, Collard CD. Vascular ischaemia and reperfusion injury. Br Med Bull 2004;70:71-86.

6. Wall CJ, Santamaria J. Extracorporeal membrane oxygenation: an unusual cause of acute limb compartment syndrome. Anaesth Intensive Care 2010;38:5602.

7. Percival TJ, Rasmussen TE. Reperfusion strategies in the management of extremity vascular injury with ischaemia. Br J Surg 2012;99 Suppl 1:66-74.

8. Reffelmann T, Hale SL, Dow JS, Kloner RA. No-reflow phenomenon persists long-term after ischemia/reperfusion in the rat and predicts infarct expansion. Circulation 2003;108:2911-7.

9. Park SE, Kim DY, Park ES. Hyperkalemia in a pa- 
Jin Young Go, et al.

tient with rhabdomyolysis and compartment syndrome: a case report. Korean J Anesthesiol 2010;59 Suppl:S37-40.
10. Sheridan GW, Matsen FA 3rd. Fasciotomy in the treatment of the acute compartment syndrome. J Bone Joint Surg Am 1976;58:112-5. 